\title{
Erratum to "Evaluation of a Suspended Personal Radiation Protection System vs. Conventional Apron and Shields in Clinical Interventional Procedures" [Open Journal of Radiology 3 (2013) 143-151]
}

\author{
Chet R. Rees \\ Baylor Scott and White Health, Dallas, USA \\ Email: chet.rees@bswhealth.org
}

How to cite this paper: Rees, C.R. (2017) Erratum to "Evaluation of a Suspended Personal Radiation Protection System vs. Conventional Apron and Shields in Clinical Interventional Procedures" [Open Journal of Radiology 3 (2013) 143-151]. Open Journal of Radiology, 7, 249-249.

https://doi.org/10.4236/ojrad.2017.74027

Received: May 16, 2013

Accepted: June 16, 2013

Published: June 23, 2013

Copyright $\odot 2017$ by author and Scientific Research Publishing Inc. This work is licensed under the Creative Commons Attribution International License (CC BY 4.0).

http://creativecommons.org/licenses/by/4.0/
The original online version of this article (C. Savage, T. Seale IV, C. Shaw, B. Angela, D. Marichal and C. Rees, "Evaluation of a Suspended Personal Radiation Protection System vs. Conventional Apron and Shields in Clinical Interventional Procedures," Open Journal of Radilogy, 3, 143-151.

http://dx.doi.org/10.4236/ojrad.2013.33024) was mistaken of the total isolates number in Table 2. The authors wish to correct the errors to: Table 2. Other

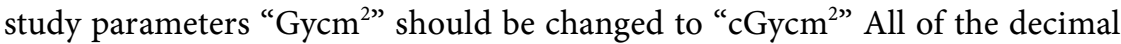
points (“.”) should be changed to commas (“””). For example, "47.486” should be " 47,486 ".

Table 2. Other study parameters.

\begin{tabular}{|c|c|c|c|c|c|c|}
\hline & \multirow{2}{*}{$\begin{array}{c}\text { Dosimeter } \\
\text { Type }\end{array}$} & \multirow{2}{*}{$\begin{array}{l}\text { Procedures } \\
\quad(\mathrm{N})\end{array}$} & \multirow{2}{*}{$\begin{array}{c}\text { Operators } \\
(\mathrm{N})\end{array}$} & \multirow{2}{*}{$\begin{array}{l}\text { Minutes } \\
\text { Fluoroscopy }\end{array}$} & \multicolumn{2}{|c|}{ Patient DAP $\left(\mathrm{cGy} \mathrm{cm}^{2}\right)$} \\
\hline & & & & & DAP Fluoroscopy & DAP Total \\
\hline Phase I & OSL badges & 67 & & & & \\
\hline Zgrav & & 32 & 3 & 307 & $47,486^{*}$ & 267,801 \\
\hline LAS & & 35 & 3 & 307 & $50,561^{* *}$ & 318,839 \\
\hline Phase II & EDD-30 & & & & & \\
\hline Eye & & 50 & & & & \\
\hline Zgrav & & 28 & 2 & 329 & 103,884 & 281,364 \\
\hline LAS & & 22 & 3 & 122 & 47,734 & 222,364 \\
\hline Wrist & & 21 & & & & \\
\hline Zgrav & & 15 & 2 & 186 & 83,316 & 414,680 \\
\hline LAS & & 7 & 2 & 40 & 22,083 & 131,520 \\
\hline
\end{tabular}

"Data available for last 13 cases, 112 minutes of fluoroscopy. " Data available for last 14 cases, 132 minutes fluoroscopy. 